Revista de la Escuela de Ciencias de la Educación, año 14, nRo. 13, vol. 1, enero a junio de 2018. Páginas 105-126. ISSN 1851-6297 (DESDE DICIEMBRE DE 2006 A DICIEMBRE DE 2017). ISSN 2362-3349 (EN LiNEA). PARA UN ABORDAJE TRANSVERSAL DE LA ESI EN EL ESPACIO CURRICULAR DE FORMACIÓN ÉTICA Y CIUDADANA: REFLEXIONES SURGIDAS DESDE EL TRABAJO en formación docente. Melina Mailhou y Virginia Sabao Dominguez.

\title{
PARA UN ABORDAJE TRANSVERSAL DE LA ESI EN EL ESPACIO CURRICULAR DE FORMACIÓN ÉTICA Y CIUDADANA: REFLEXIONES SURGIDAS DESDE EL TRABAJO EN FORMACIÓN DOCENTE
}

\author{
Por Melina Mailhou* \\ Universidad Nacional de Rosario, Argentina \\ melinamailhou@gmail.com \\ Virginia Sabao Domínguez \\ Universidad Nacional de Rosario, Argentina \\ mvsabao@yahoo.com.ar
}

Recibido: 20/09/2017 Aceptado: 04/12/2017

\begin{abstract}
“¿Estaríamos ya liberados de esos dos largos siglos donde la historia de la sexualidad debería leerse en primer término como la crónica de una represión creciente?

(...) no es posible liberarse sino a un precio considerable: haría falta nada menos que una transgresión de las leyes, una anulación de las prohibiciones, una irrupción de la palabra, una restitución del placer a lo real y toda una nueva economía en los mecanismos del poder; pues el menor fragmento de verdad está sujeto a condición política." (Foucault, 2000, p. 11).
\end{abstract}

* Es Profesora de Filosofía por la Universidad Nacional de Rosario. Actualmente se encuentra realizando la Licenciatura en Filosofía en la misma casa de estudios y la Diplomatura en Pensamiento Descolonial en la Universidad Nacional de Jujuy.

** Es Profesora y Licenciada en Antropología por la Universidad Nacional de Rosario. Actualmente se encuentra realizando el Doctorado en Humanidades y Artes (orientación Antropología) dictado por la Universidad Nacional de Rosario. Maestranda en DDHHUniversidad Nacional de Rosario. 
Revista de la Escuela de Ciencias de la Educación, año 14, nRo. 13, vol. 1, enero a junio de 2018. Páginas 105-126. ISSN 1851-6297 (DESDE DICIEMBRE DE 2006 A DICIEMBRE DE 2017). ISSN 2362-3349 (EN LiNEA). PARA UN ABORDAJE TRANSVERSAL DE LA ESI EN EL ESPACIO CURRICULAR DE FORMACIÓN ÉTICA Y CIUDADANA: REFLEXIONES SURGIDAS DESDE EL TRABAJO en formación docente. Melina Mailhou y Virginia Sabao Dominguez.

\section{Resumen}

Este trabajo quiere pensar las articulaciones entre los Lineamientos Curriculares de la Educación Sexual Integral (ESI) como contenido transversal, y el espacio curricular Formación Ética y Ciudadana (FEyC) en el Ciclo Básico de la Educación Secundaria. Para ello, se propone un recorrido por el Diseño Curricular de Educación Secundaria Orientada de Santa Fe (DCESO) en aspectos referidos a la ESI y a la formación en ciudadanía. El objetivo es rastrear cómo, desde el 2008 hasta el presente, a nivel Nacional y Jurisdiccional, se fueron configurando y reconfigurando las problemáticas; cómo se volvieron hegemónicos los distintos discursos disciplinares al interior de este corpus, y de qué modo, en definitiva, se concretó la multidisciplinariedad, aunque no una perspectiva transdisciplinaria, interdisciplinaria y transversal. Estas reflexiones surgen del trabajo en la Formación Docente en profesorados de nivel superior (universitario y no universitario).

Palabras clave: Sexualidad - Ciudadanía - Transversalización - Multidisciplinariedad - Transdisciplinariedad.

\section{Abstract}

In this work we want to think about the articulations between the Curricular Guidelines of the Integral Sexual Education (ESI) considered as transversal content, and the curricular space Ethical and Citizenship Training (FEyC) in the Basic Cycle of Secondary Education. To do this, we propose a tour of the Curricular Design of Oriented Secondary Education of Santa Fe (DCESO) in aspects related to $\mathrm{ESI}$ and citizenship training. The objective is to trace how, from 2008 to the present, at a National and Jurisdictional level, problems were being configured and reconfigured; how the different disciplinary discourses within this corpus became hegemonic, and how, ultimately, multidisciplinarity was concretized, although not a transdisciplinary, interdisciplinary and transversal perspective. These reflections arise from the work in the Teacher Training in teachers of higher level (university and non-university).

Keywords: Sexuality - Citizenship - Transversalization - MultidisciplinarityTransdisciplinarity.

\section{Introducción}

El objetivo de este trabajo es problematizar el modo en que se producen las articulaciones entre los lineamientos curriculares del Programa Nacional de Educación Sexual Integral (ESI) y el espacio curricular Formación Ética y Ciudadana (FEyC) en el Ciclo Básico de la Educación Secundaria. Estos lineamientos curriculares elaborados por el Ministerio de Educación a partir de 
Revista de la Escuela de Ciencias de la Educación, año 14, nRo. 13, vol. 1, enero a junio de 2018. Páginas 105-126. ISSN 1851-6297 (DESDE DICIEMBRE DE 2006 A DICIEMBRE DE 2017). ISSN 2362-3349 (EN LINEA). PARA UN ABORDAJE TRANSVERSAL DE LA ESI EN EL ESPACIO CURRICULAR DE FORMACIÓN ÉTICA Y CIUDADANA: REFLEXIONES SURGIDAS DESDE EL TRABAJO en formación docente. Melina Mailhou y Virginia Sabao Dominguez.

la sanción de la Ley Nacional 26.150, se establecen como contenidos transversales que, por tanto, deben articularse con todas las asignaturas.

Consideramos que el espacio curricular FEyC, pensado desde el DCESO como destinado a "proponer prácticas sociales orientadas hacia la libertad responsable y el respeto por el otro en un contexto democrático" (DCESO, Santa Fe, 2014, p. 16), se presenta en sí mismo como potencialmente privilegiado para trabajar los contenidos de la ESI, por tanto, profundizar en las reflexiones sobre el sentido que debería imprimírsele a la articulación de los contenidos procedentes de ambos espacios, resulta una tarea ineludible.

Partimos de observar que las "recomendaciones para la transversalización de la ESI" propuestas por el DCESO son realmente muy escuetas y de escaso valor orientativo. Las mismas se limitan al siguiente párrafo:

La apropiación del enfoque de los derechos humanos implica visibilizar las luchas y la movilización de aquellos sujetos que no fueron incluidos en la Declaración Universal de los Derechos Humanos de 1948. Es necesario priorizar la Convención para la eliminación de todas las formas de discriminación contra las mujeres (CEDAW, 1979) porque da cuenta de la vigilancia permanente al carácter androcéntrico del campo jurídico, entre otros, constituyendo un punto de partida necesario para entender la sexualidad en el terreno de los derechos sexuales y reproductivos y una vida libre de violencia. (DCESO, Provincia de Santa Fe, 2014, p. 112, la negrita es nuestra).

Así, se observa que la sugerencia para la construcción del enfoque de ESI para FEyC en el DCESO, tiene la particularidad de acotar la mirada privilegiando una única perspectiva disciplinar: el campo jurídico, por lo que la "integralidad" desde la que debería enfocarse la educación sexual, no estaría garantizándose. A su vez, la perspectiva multidisciplinar que se enfatiza en los NAP (1) y el DCESO en relación al espacio de FEyC no logra concreción efectiva ya que los enfoques jurídico-normativo y moralizante son los predominantes. Por el contrario, se replica una perspectiva reductiva respecto del área curricular mencionada, que la circunscribe al ámbito de la formación cívica y el análisis jurídico exclusivamente.

Por otra parte, si bien el DCESO, en consonancia con el criterio acordado por el Consejo Federal de Educación (CFE), prescribe la transversalidad de los contenidos de la ESI, no hay en el documento un desarrollo en profundidad que explicite cómo debería concretarse una transversalidad que en ningún momento es definida. De este modo, al no estar explícitamente enunciados entre los contenidos de las asignaturas y al ser tan poco específico lo que se recomienda como articulación, la presencia del Programa Nacional de ESI, se desdibuja notablemente. 
Revista de la Escuela de Ciencias de la Educación, año 14, nRo. 13, vol. 1, enero a junio de 2018. Páginas 105-126. ISSN 1851-6297 (DESDE DICIEMBRE DE 2006 A DICIEMBRE DE 2017). ISSN 2362-3349 (EN LiNEA). PARA UN ABORDAJE TRANSVERSAL DE LA ESI EN EL ESPACIO CURRICULAR DE FORMACIÓN ÉTICA Y CIUDADANA: REFLEXIONES SURGIDAS DESDE EL TRABAJO en formación docente. Melina Mailhou y Virginia Sabao Dominguez.

Los Lineamientos Curriculares propuestos a partir de la promulgación de la Ley 26.150 de ESI, sostienen una voluntad de trascender el paradigma biologicista según el cual tradicionalmente se ha abordado la educación sexual; concibiendo por el contrario, su enseñanza en las instituciones educativas, como la destinada a situar la cuestión de la sexualidad y la construcción de las identidades en el orden complejo de lo sociocultural, pensándola así desde una perspectiva integral. Es de acuerdo con esta complejidad, que la Ley propone que el abordaje de los contenidos de la ESI debe ser no sólo transversal sino también interdisciplinario. Todo docente, independientemente de cuál sea su campo disciplinar específico, debería enseñar los contenidos, y plantear las articulaciones de los mismos con tópicos de su propio campo, así como también con los de otros espacios curriculares. En este sentido, abordar los contenidos de la ESI supone necesariamente llevar a cabo tanto una revisión de la propia formación recibida (al menos para los casos de los profesorados universitarios en antropología y filosofía), como de las propuestas estatales sobre el tema, desde una mirada analítico/crítica. (2)

EI DCESO del Ministerio de Educación de Santa Fe (2014), en consonancia con la mencionada Ley, define la sexualidad como

una dimensión constitutiva de la condición humana que se expresa en sentimientos, pensamientos y prácticas concretas, concepción despegada de la mera genitalidad, que requiere un abordaje transversal para poder comprenderla y explicarla. Necesita de las herramientas analíticas de todos los campos disciplinares para reconocer formas y mecanismos de producción de prácticas estereotipadas, androcéntricas y heteronormativas con miras a su transformación. (p. 111).

En función de pensar a la sexualidad como constitutiva de la condición humana, entendiendo que los que enseñamos y aprendemos somos en cada caso y en todo momento sujetos inscritos en procesos identitarios, la ESI se propone principalmente (aunque no exclusivamente), como un contenido transversal. Tal como se sostiene desde el Ministerio de Educación de la Nación en los Lineamientos Curriculares para la ESI (2008):

la transversalidad y la existencia de un espacio curricular específico no constituyen alternativas excluyentes. Ambas pueden coexistir en cada establecimiento en la educación primaria y secundaria. Sin embargo, y en relación con la ESI, sería recomendable organizar espacios transversales de formación desde la educación inicial y primaria, para luego considerar la apertura en la educación secundaria de espacios específicos, que puedan formar parte de asignaturas ya existentes en el currículo, o de nuevos espacios a incorporar. No debería entenderse que la existencia de un espacio específico implica abandonar la preocupación por el abordaje interdisciplinario de la ESI. (p.14). 
Revista de la Escuela de Ciencias de la Educación, año 14, nRo. 13, vol. 1, enero a junio de 2018. Páginas 105-126. ISSN 1851-6297 (DESDE DICIEMBRE DE 2006 A DICIEMBRE DE 2017). ISSN 2362-3349 (EN LINEA). PARA UN ABORDAJE TRANSVERSAL DE LA ESI EN EL ESPACIO CURRICULAR DE FORMACIÓN ÉTICA Y CIUDADANA: REFLEXIONES SURGIDAS DESDE EL TRABAJO en formación docente. Melina Mailhou y Virginia Sabao Dominguez.

Garantizar el tratamiento de la ESI en forma transversal, interdisciplinaria, sistemática y continua, evitando que sus propósitos formativos se diluyan, implica necesariamente que en el diseño de las planificaciones para el aula, los contenidos de la ESI asuman un lugar concreto. En favor de llegar a hacer efectiva la transversalidad, el objetivo general que nos planteamos es el de reflexionar sobre las posibilidades de una articulación entre los ejes que propone la ESI y el espacio curricular de Formación Ética y Ciudadana, en el Ciclo Básico.(3)

\section{La constitución de la Ley y el Programa de ESI: ambivalencias, tensiones, contradicciones}

La Ley Nacional de ESI, se sanciona en el año 2006 en Argentina, prescribiendo la obligatoriedad de la educación sexual integral en todos los niveles de enseñanza. Consecuentemente se crea el Programa Nacional de ESI para dar forma a la implementación de la ley en todo el territorio nacional. Tal como indica Bilinkis (2013), es interesante conocer el proceso que da como resultado la sanción de la Ley Nacional de ESI a fin de interpretar las controversias establecidas:

el proceso que dio como resultado la sanción de la Ley Nacional de Educación Sexual Integral estuvo acompañado de fuertes disputas y demandas que enfrentaron a diversos actores (partidos políticos, organizaciones de la sociedad civil, iglesia católica y otros credos, entre otros) con perspectivas heterogéneas tanto en torno a la definición de lo que se concibe como sexualidad y educación sexual, así como también en lo que respecta a la edad de inicio en la formación en educación sexual y la competencia del Estado y las familias en esa área de enseñanza. (Bilinkis, 2013, p. 28).

Así, en la investigación en curso que Bilinkis (2013) emprende, se intentan distinguir algunas consideraciones generales sobre los debates parlamentarios que nos permiten reconocer en la normativa sobre ESI, el resultado sintético de contradicciones históricas presentes en la enseñanza formal y no formal de estos contenidos. Entre estas tensiones, cabe mencionar la que surge a partir de concebir a la educación sexual como una cuestión privada; implicando así no solo una reificación de la distinción público/privado, sino una invisibilización de la dimensión pública de la sexualidad y un desdibujamiento de la práctica histórica escolar en educación sexual, al ser concebida como un eje no explícito de enseñanza, volviendo difusas las prácticas condensadas hasta el presente por las escuelas. (Bilinkis, 2013, p. 29).

Asimismo, se observa que los debates parlamentarios definieron unos contenidos "científicos" en torno a la educación sexual como opuestos a la educación sexual familiar entendida como constituida por contenidos valorativos/irracionales, intentando posicionar a la escuela como único ámbito de 
Revista de la Escuela de Ciencias de la Educación, año 14, nRo. 13, vol. 1, enero a junio de 2018. Páginas 105-126. ISSN 1851-6297 (DESDE DICIEMBRE DE 2006 A DICIEMBRE DE 2017). ISSN 2362-3349 (EN LiNEA). PARA UN ABORDAJE TRANSVERSAL DE LA ESI EN EL ESPACIO CURRICULAR DE FORMACIÓN ÉTICA Y CIUDADANA: REFLEXIONES SURGIDAS DESDE EL TRABAJO en formación docente. Melina Mailhou y Virginia Sabao Dominguez.

saber legítimo y por tanto enfrentado a los del saber popular o cotidiano. Así aparecería una intención legitimadora de la función del Estado en la educación sexual, por la tarea desmitificadora de la enseñanza formal, estableciendo un consenso en torno a la idea de vulnerabilidad y riesgo para la definición de la condición de los niños/niñas,

articulando discursos y percepciones disímiles sobre "problemáticas sociales" relativas a los/as niños/as ("acoso", "abuso sexual infantil”, "sobreinformación" y "falta de información veraz" sobre la sexualidad, etc.) que los/ as situaron como "vulnerables" y "en riesgo".

Se buscó poner en tensión la tendencia de la opinión pública a relacionar la educación sexual en la niñez con la estimulación temprana de las relaciones sexuales y la "pérdida de la inocencia infantil", la ley pareciera presentarse como un acto de expropiación de ciertos saberes sobre la sexualidad que circulan por distintos ámbitos, sea el de los medios de comunicación o el familiar. Este acto de expropiación basado en un fuerte componente racional y de cientificidad contribuye a situar a las escuelas como instituciones en las cuales tiene lugar una "transmisión de conocimientos verdaderos", en contraposición a la "formación en valores" que llevan adelante las familias. (Bilinkis, 2013, p. 28).

Estas jerarquizaciones de temas y problemas que se detectan en el debate parlamentario, se observarán luego, con efecto de arrastre, en las propuestas de Lineamientos Curriculares de ESI, aunque allí aparecen ciertas complementaciones y adaptaciones que tensionan la primera tendencia constituida por parte de los parlamentarios.

Tal como se dijo, a fin de favorecer la implementación de la Ley, se crea el Programa Nacional de ESI y se elaboran los Lineamientos Curriculares. En este sentido resulta valioso acudir a un trabajo en el que se presentan impresiones de funcionarios/as estatales y de organizaciones sociales de intervención educativa que participaron del proceso de implementación del programa. Consideramos fundamental reconocer en los diseñadores de los Lineamientos Curriculares ESI, intereses, intenciones y expectativas colocadas respecto de las posibilidades de apropiación de la ESI por parte de los agentes sociales y escolares en particular.

A continuación se reproducen fragmentos de una entrevista realizada a Mirta Marina, Coordinadora del Programa Nacional ESI (2012).

¿Por qué, a diferencia de lo que pasa con el resto de los contenidos, la educación sexual merece una ley específica?

...El antecedente más directo, en el nivel nacional, es la Ley de Salud Sexual y Procreación Responsable, que ya decía que los docentes tenían la responsabilidad de informar. Pero creo que fue necesaria una ley específica porque la escuela no asumía esa tarea. La batalla de generar una ley 
Revista de la Escuela de Ciencias de la Educación, año 14, nRo. 13, vol. 1, enero a junio de 2018. Páginas 105-126. ISSN 1851-6297 (DESDE DICIEMBRE DE 2006 A DICIEMBRE DE 2017). ISSN 2362-3349 (EN LiNEA). PARA UN ABORDAJE TRANSVERSAL DE LA ESI EN EL ESPACIO CURRICULAR DE FORMACIÓN ÉTICA Y CIUDADANA: REFLEXIONES SURGIDAS DESDE EL TRABAJO en formación docente. Melina Mailhou y Virginia Sabao Dominguez.

específica tiene que ver con la presión de distintos colectivos interesados en que esto realmente ocurra, como las organizaciones representantes de la diversidad sexual, los colectivos feministas que aportaron el enfoque de género y distintas agrupaciones del campo de la promoción de la salud, que vienen luchando desde hace varios años para que la educación sexual sea un contenido curricular que permita mejorar la calidad de vida de las chicas y de los chicos. (Fuente: Entrevista realizada a Mirta Marina. Entrevistadores: Diego Herrera- Diego Rosemberg. 2012. p. 12).

Ya en este fragmento de entrevista se pueden reconocer condiciones previas y tensiones existentes en torno a la cuestión de la ESI. La precondición normativa o antecedente de ESI, resulta un condicionante de la práctica de carácter sustantivo, ya que la ley que antecede, es la Ley de Salud sexual y Procreación responsable. Así, es posible entender ciertos sesgos o límites que impone esta primera normativización de la cuestión de la sexualidad en la reconstitución normativa que implica la propuesta de ESI. Es decir que, como se observa, la lógica normativa precedente se centra en el enfoque higienista, sanitarista o médico. Siendo éste el antecedente de la constitución de la ESI, el enfoque género y diversidad sexual, ingresa tensionándose con la perspectiva de medicalización en torno a la sexualidad ya existente en la ley que se invoca como antecedente directo.

A su vez, es interesante observar cómo se justifica la legitimidad de la creación de la ESI, la funcionaria incluye dos fenómenos convergentes: el corrimiento de la escuela respecto a asumir su responsabilidad en la educación sexual desde una mirada integral, y la presión de organizaciones sociales (LGBT y feministas). Queda así claramente explicitado un sistema de condiciones tensionales en las que, por un lado ciertos grupos buscan la visibilización de la cuestión de la sexualidad y la constitución de identidades en torno a ella, que por su parte requiere de un abordaje multidisciplinario y por lo tanto científico (desde diversas perspectivas académicas y enfatizando el enfoque de género). Mientras que, por otro lado las instituciones escolares tienden a asumir la cuestión desde el enfoque de la medicalización (que adquiere mayor consonancia con la ley de Salud sexual y procreación responsable que es reconocida como antecedente de la 26.150-ESI) o bien a rechazar la idea de una responsabilidad en torno a la educación sexual.

A su vez, es posible reconocer en el discurso de la funcionaria, una serie de temas centrales a tener en cuenta en el enfoque de ESI:

¿Cuáles son los contenidos que propone el Ministerio?

Se trata de ejes que atraviesan desde el jardín hasta el secundario. Por mencionar uno: el cuidado del propio cuerpo y el del otro o la otra.

...por ejemplo, es poder resistir presiones de pares o de adultos, poder decir que no cuando algo incomoda. También es conocer sobre métodos 
Revista de la Escuela de Ciencias de la Educación, año 14, nRo. 13, vol. 1, enero a junio de 2018. Páginas 105-126. ISSN 1851-6297 (DESDE DICIEMBRE DE 2006 A DICIEMBRE DE 2017). ISSN 2362-3349 (EN LiNEA). PARA UN ABORDAJE TRANSVERSAL DE LA ESI EN EL ESPACIO CURRICULAR DE FORMACIÓN ÉTICA Y CIUDADANA: REFLEXIONES SURGIDAS DESDE EL TRABAJO en formación docente. Melina Mailhou y Virginia Sabao Domínguez.

anticonceptivos. Ese es un contenido; otra línea es la equidad de género. Desde la más tierna infancia proponemos que no hay unas actividades para varones y otras para mujeres. Se trata de formar una sociedad más igualitaria, porque donde hay violencia de género subyace la inferiorización de las mujeres.

...Otro gran eje es la expresión de los sentimientos, el gran ausente en otras miradas de la educación sexual que son más biologicistas o que sólo apuntan a la salud sexual y reproductiva.

...se puede abordar el tema del deseo del otro como límite a la expresión de sentimientos. Además, hay muchas cuestiones que para la mirada tradicional no entrarían dentro de la educación sexual, como el análisis crítico de los modelos de belleza que muestran las publicidades o los programas de televisión. (Fuente: Entrevista realizada a Mirta Marina. Entrevistadores: Diego Herrera- Diego Rosemberg. 2012. pp.14-15).

En este contexto, nos interesa comenzar a vislumbrar la cuestión de la transversalidad y la multidisciplinariedad, ya que, tal como aparece retratado el enfoque de ESI en la expresión anterior, se observa una muy poco lograda mirada integral para pasar a constituir una propuesta de tipo sumativa dónde diversos abordajes: bio-médico, jurídico, psicológico o comunicacional aparecen meramente adicionados en una secuencia que no presenta articulaciones claras entre los mismos(4). En definitiva, la articulación de miradas disciplinares no se efectiviza, mientras se enfatiza la importancia de un abordaje científico del problema que tiende a presentarse como contrapuesto a las concepciones que constituimos en torno a él en la vida cotidiana. Así la ESI ingresa como contenido escolar, bajo la lógica legitimante de la racionalidad científica entendida con una función desmitificadora de saberes anteriores y exteriores a la escuela. En tal sentido, y a partir del reconocimiento de ese sesgo, concebimos que la transdisciplinariedad sería la estrategia más adecuada a este campo de indagación, tal como sucede con el campo de los derechos humanos que deben problematizarse en el espacio de FEyC.

\section{Formación Ética y Ciudadana en los Lineamientos Curriculares para la Educación Sexual Integral}

Como hemos venido destacando, partir de la aprobación de la Ley 26.150 queda establecido como propósito primordial la responsabilidad estatal de validar el derecho de niños, niñas y jóvenes a recibir Educación Sexual Integral en todos los establecimientos educativos públicos de gestión estatal y privada. Dentro de este marco político y constitucional se crean los Lineamientos Curriculares de ESI como primer nivel de desarrollo curricular en relación con la Educación Sexual, base a partir de la cual las distintas jurisdicciones realizarán las correspondientes adecuaciones de acuerdo a las propias realidades socioculturales. A su vez, dentro de este marco jurisdiccional, cada institución 
Revista de la Escuela de Ciencias de la Educación, año 14, nRo. 13, vol. 1, enero a junio de 2018. Páginas 105-126. ISSN 1851-6297 (DESDE DICIEMBRE DE 2006 A DICIEMBRE DE 2017). ISSN 2362-3349 (EN LiNEA). PARA UN ABORDAJE TRANSVERSAL DE LA ESI EN EL ESPACIO CURRICULAR DE FORMACIÓN ÉTICA Y CIUDADANA: REFLEXIONES SURGIDAS DESDE EL TRABAJO en formación docente. Melina Mailhou y Virginia Sabao Dominguez.

educativa deberá proponer sus propios desarrollos curriculares en consonancia con su propia situacionalidad, siempre dentro de los marcos, objetivos y pautas definidos por la Ley.

Los propósitos formativos de estos Lineamientos Curriculares, se sostienen en la necesidad de: ampliar el horizonte para el desarrollo pleno de la subjetividad de los adolescentes, reconociendo derechos y responsabilidades propios y de los otros; expresar y valorar emociones y sentimientos presentes en las relaciones humanas en relación con la sexualidad, respetando y haciendo respetar los derechos humanos; propiciar el conocimiento del cuerpo humano promoviendo hábitos de cuidado y promoción de la salud sexual y derechos reproductivos; promover una educación en valores como: la solidaridad, el amor, el respeto a la intimidad propia y ajena, el respeto a la vida y la integridad de las personas; promover aprendizajes basados en el respeto a la diversidad y el rechazo a toda forma de discriminación; desarrollar competencias para la resolución de conflictos en base al diálogo (Lineamientos Curriculares para la ESI, 2008, pp.13-14).

Es decir que, de acuerdo con lo expuesto, la ESI pretende sacar a la sexualidad de la mera reducción a la reproducción biológica y de una mirada moralizante, colocándola en el orden complejo de lo construido socioculturalmente. Sintetizando lo anterior resaltamos los cinco ejes claves que estructuran a la ESI:

1) La ESI es un derecho.

2) La ESI debe desarrollarse desde una perspectiva de género.

3) La ESI contempla la diversidad.

4) La ESI integra la afectividad.

5) La ESI enfoca la cuestión del cuidado del cuerpo y de la salud (siempre desde una perspectiva de los derechos).

Delineados estos ejes, la Ley asume que todo/a docente debe ser idóneo para enseñar los contenidos de modo transversal, articulándolos con los de su propio campo disciplinar. Veamos entonces a continuación algunos de los contenidos que proponen los Lineamientos Curriculares para la ESI para el espacio curricular Formación Ética y Ciudadana, Ciclo Básico (2008, pp. 3435):

La construcción cooperativa de normas a partir del diálogo sobre situaciones cotidianas que ocurren en el aula y en la escuela, que manifiestan prejuicios contra varones y/o mujeres y/o deterioran las relaciones interpersonales, en lo que refiere al respeto, cuidado de sí mismo y de los otros/as. El reconocimiento y expresión de los deseos y necesidades propios y el respeto de los deseos y las necesidades de los/as otros/as, en el marco del respeto a los derechos humanos.

El conocimiento y la aceptación de las normas que involucran la propia integridad física y psíquica en el ámbito de las relaciones afectivas y sexuales. 
Revista de la Escuela de Ciencias de la Educación, año 14, nRo. 13, vol. 1, enero a junio de 2018. Páginas 105-126. ISSN 1851-6297 (DESDE DICIEMBRE DE 2006 A DICIEMBRE DE 2017). ISSN 2362-3349 (EN LíNEA). PARA UN ABORDAJE TRANSVERSAL DE LA ESI EN EL ESPACIO CURRICULAR DE FORMACIÓN ÉTICA Y CIUDADANA: REFLEXIONES SURGIDAS DESDE EL TRABAJO en formación docente. Melina Mailhou y Virginia Sabao Domínguez.

El reconocimiento y la reflexión sobre situaciones de violencia en las relaciones interpersonales (específicamente afectivas y sexuales) o sobre conductas de imposición sobre los derechos de otros/as.

El conocimiento de leyes, tratados y convenios nacionales e internacionales relativos a los derechos humanos en general y de los niños, niñas y adolescentes relacionados con la salud, la educación y la sexualidad y el desarrollo de competencias relacionadas con la exigibilidad de estos derechos.

El conocimiento de los derechos de las diversidades sexuales y de la responsabilidad del Estado frente a situaciones de discriminación y violación de derechos. (Lineamientos Curriculares para la ESI, 2008, p. 35).

Ahora bien, aun cuando estos propósitos formativos queden, no vemos que los mismos se reflejen de manera contundente en otros niveles de diseño curricular. (5).

Ya sea en los Núcleos de Aprendizajes Prioritarios (aprobados por Resolución CFE Nro. 141/11), como en el DCESO del 2014 no se desarrolla una propuesta de articulación de los contenidos de la ESI con los de la propia FEyC que haga hincapié en el carácter multidisciplinario o interdisciplinario de ambos espacios curriculares.

\section{Formación Ética y Ciudadana en el Diseño Curricular de Educación Secundaria Orientada de la Provincia de Santa Fe}

En la jurisdicción de Santa Fe se observa que se han establecido contenidos para el área de FEyC que reproducen las orientaciones del CFE. En el primer ciclo se distinguen cuatro ejes de contenidos en la presentación del espacio curricular. Para primer año se establecen los siguientes ejes: En relación con la reflexión ética, En relación con los Derechos Humanos y los Derechos de los Niños y Adolescentes, En relación con las identidades y las diversidades, En relación con la organización social y una ciudadanía participativa. Y en segundo año se replican los primeros tres ejes mencionados para el primero y se agrega un cuarto eje: En relación con la organización social y una ciudadanía responsable.

\section{Algunas apreciaciones acerca de la fundamentación}

A continuación analizaremos la composición de la fundamentación, las consideraciones metodológicas y los ejes de trabajo, a fin de identificar cómo aparecen allí los contenidos que podrían relacionarse con la ESI.

En la fundamentación se puede ver que el DCESO establece aclaraciones que nos permiten identificar algunas particularidades en la propuesta:

En primer lugar se observa una definición de la ciudadanía como práctica ética, entendiendo a la Ética como el ámbito de conocimiento desde el cual considerarla. Así, se propone una hegemonía de la subdisciplina de la Ética 
Revista de la Escuela de Ciencias de la Educación, año 14, nRo. 13, vol. 1, enero a junio de 2018. Páginas 105-126. ISSN 1851-6297 (DESDE DICIEMBRE DE 2006 A DICIEMBRE DE 2017). ISSN 2362-3349 (EN LiNEA). PARA UN ABORDAJE TRANSVERSAL DE LA ESI EN EL ESPACIO CURRICULAR DE FORMACIÓN ÉTICA Y CIUDADANA: REFLEXIONES SURGIDAS DESDE EL TRABAJO en formación docente. Melina Mailhou y Virginia Sabao Dominguez.

dentro del campo del saber filosófico para el abordaje del ethos que se concibe como el objeto exclusivo de análisis de la misma. Y dentro del campo de la Ética se toma posición respecto a una línea de delimitación que refiere a la reflexión ética como aquella que permite distinguir entre el bien y el mal. (6).

Sin embargo, ya tempranamente en el campo de la antropología y desde el punto de vista del relativismo cultural, es posible reconocer una necesaria diferenciación entre absolutos y universales. Así, la moral constituiría un universal, aunque las diversas formas que adopta son productos de la particular experiencia histórica de las sociedades concretas. (Boas, 1964; Herskovits, 1968; Mead, 1973; Kluckhohn, 1981).

En cada sociedad y/o grupo los criterios están sujetos a continuas interrogaciones y a continuos cambios. (Herskovits, 1968, p. 91).

\section{No hay muchas culturas, por ejemplo, en las que se insista en una rígida separación entre el bien y el mal, tal como nosotros la hemos establecido. \\ En una cultura donde se exaltan los valores absolutos, el relativismo de un mundo que abarca muchos modos de vida será difícil de comprender. Más bien ofrecerá un terreno para juicios de valor cuya base es la proporción en que un cuerpo dado de costumbres se asemeja o difiere de los propios de la cultura euroamericana. (Herskovits, 1968, pp. 92-93).}

Así, se observa cómo las concepciones acerca de una ética dialógica que propone el NAP, se ven tensionadas en la propuesta jurisdiccional debido a la inclusión de una perspectiva sobre la Ética que se inscribe claramente en definiciones judeo-cristianas sobre el discernimiento moral. (7).

En segundo lugar, se presenta una mirada sobre el diálogo que lo coloca claramente como una mediación para el consenso o resolución de conflictos, invisibilizando la función que este mismo tiene en la identificación de los disensos. Esta perspectiva consensual, coloca al diálogo sólo como un medio para la realización de acuerdos minusvalorando la función que éste tiene en la determinación de los conflictos como elementos no meramente pasajeros en la constitución social, sino como componentes estructurantes de la sociabilidad, posición cercana a la propuesta por Habermas (2005) en torno a la política en tanto deliberativa.

Esta perspectiva consensual incluso se encuentra en tensión con las recomendaciones del Documento de Desarrollo Curricular para la Educación Primaria y Secundaria: Núcleos Interdisciplinarios de Contenidos. La educación en acontecimientos. (Ministerio de Educación de la Pcia. de Sta. Fe, 2016). En los NIC de 2016, se indica una perspectiva acerca de la política que recupera los aportes de Mouffe (2014), quien critica abiertamente la perspectiva de Habermas (2005) acerca de la política deliberativa, oponiéndole una concepción 'agonística' o 'radical' sobre la política y la democracia. El aporte de la autora 
Revista de la Escuela de Ciencias de la Educación, año 14, nRo. 13, vol. 1, enero a junio de 2018. Páginas 105-126. ISSN 1851-6297 (DESDE DICIEMBRE DE 2006 A DICIEMBRE DE 2017). ISSN 2362-3349 (EN LiNEA). PARA UN ABORDAJE TRANSVERSAL DE LA ESI EN EL ESPACIO CURRICULAR DE FORMACIÓN ÉTICA Y CIUDADANA: REFLEXIONES SURGIDAS DESDE EL TRABAJO en formación docente. Melina Mailhou y Virginia Sabao Dominguez.

consiste precisamente en inscribir a la política en el orden del conflicto y el 'agonismo'. De esta manera afirma que:

(...) sólo cuando reconocemos lo político en su dimensión antagónica es posible plantear la cuestión central de la política democrática. Esta cuestión, mal que les pese a los teóricos liberales, no es como negociar un acuerdo entre intereses enfrentados, ni como llegar a un acuerdo racional -es decir, totalmente inclusivo, sin ninguna exclusión- A pesar de lo que muchos liberales quieren creer, la especificidad de la política democrática no es la superación de la oposición nosotros/ellos, sino la forma diferente en que ésta se establece.

La tarea principal de una política democrática no es eliminar las pasiones ni relegarlas a la esfera de lo privado con el fin de establecer un consenso racional en la esfera pública. Por el contrario, consiste en sublimar dichas pasiones movilizadas hacia proyectos democráticos mediante la creación de formas colectivas de identificación en torno a objetivos democráticos. (Mouffe, 2014, p.28 en NIC 2016). (Fuente: Núcleos Interdisciplinarios de Contenidos. La educación en acontecimientos. Documento de Desarrollo Curricular para la Educación Primaria y Secundaria. Ministerio de Educación de la Pcia. de Sta. Fe. 2016, p.28).

En síntesis, creemos que el uso que, en la Fundamentación del DCESO, se hace de conceptos tales como "ética", "valores", "comunidad", apunta a una visión edulcorada de la política ya que, lejos de enfatizarla como práctica en la que el conflicto se entiende como consustancial, se la piensa desde el consenso. (8). Creemos que, si hay política (y si hay ética) es justamente porque no hay de antemano consenso sino porque lo predominante es el conflicto. Como afirma Claude Lefort, el conflicto es uno de los "principios constitutivos" de la política (Lefort, 1981), y consecuentemente, de la ética. El Diseño pareciera querer borrar la preminencia del conflicto en la política, tanto así como la necesidad de fundamentación en la ética, dando en cambio lugar a una bajada acrítica de "valores". En este sentido, entendemos que los NIC (2016), emergen como un documento que permite tensionar la definición presentada en el diseño jurisdiccional, aunque entendemos que no contiene la fuerza normativa que sí posee el DCESO.

\section{Aclaración acerca de las consideraciones metodológicas}

Por su parte, en las consideraciones metodológicas, la ESI tiene una mención especial asociada a la presencia de otros ejes de conocimiento, todos ellos destinados a una elaboración de seminarios temáticos intensivos lo cual la corre de un tratamiento de carácter transversal tal como se propone en los Lineamientos Curriculares ESI a nivel nacional: 
Revista de la Escuela de Ciencias de la Educación, año 14, nRo. 13, vol. 1, enero a junio de 2018. Páginas 105-126.

ISSN 1851-6297 (DESDE DICIEMBRE DE 2006 A DICIEMBRE DE 2017). ISSN 2362-3349 (EN LiNEA). PARA UN ABORDAJE

TRANSVERSAL DE LA ESI EN EL ESPACIO CURRICULAR DE FORMACIÓN ÉTICA Y CIUDADANA: REFLEXIONES SURGIDAS DESDE EL TRABAJO en formación docente. Melina Mailhou y Virginia Sabao Dominguez.

Seminarios temáticos intensivos que propongan el desarrollo de campos de producción de saberes históricamente planteados como contenidos transversales del curriculum, tales como: Educación Sexual Integral, Educación Vial, Educación para la Salud, uso responsable de las Nuevas Tecnologías, Educación para la Convivencia y la Paz. (DCESO, 2014, p. 17).

\section{Comentarios acerca de los ejes de contenido}

a. En relación con la reflexión ética

La introducción de dilemas éticos, en el DCESO, está pensada en función del ejercicio del diálogo, pero entendiendo a tal procedimiento como mediación hacia la 'construcción de acuerdos y la resolución de conflictos'. (9). Por lo tanto se hace presente de modo central el discurso normativo-jurídico y ético-filosófico, con sus efectos de neutralización y universalización en relación a los temas del espacio curricular. (10). Por su parte la concepción presentada en los NAP, hace referencia a lo compartido y lo controversial con equivalente importancia, a fin de ser reconocidos en el ejercicio del discernimiento ético. Los valores emergerían en la propuesta provincial de modo esencialista, como entidades preexistentes al ejercicio de discernimiento ético, mientras en el nivel curricular general aparecen como derivación de un ejercicio dialógico, argumentativo e incluso confrontativo de los estudiantes en el espacio escolar. (11).

b. En relación con los derechos humanos y los derechos de niños, niñas y adolescentes.

En el presente eje la única mención que encontramos relacionada con ESI para primer año, se expresa como sigue:

La identificación y comprensión de las tensiones inherentes a la defensa de los Derechos Humanos en relación con tradiciones culturales vinculadas con el cuidado del ambiente, el género y la orientación sexual... (DCESO, 2014, p. 18).

Para el segundo año y hacia el final, se incluye el siguiente ítem de contenido:

La comprensión y el análisis de los alcances legales que poseen las normativas vigentes sobre los derechos de los niños, adolescentes y jóvenes, y sus aplicaciones en situaciones conflictivas de la vida cotidiana o en casos de vulneración, tales como el maltrato infantil, abuso sexual, trata de personas. (DCESO, 2014, p. 17).

Esta aparición de la cuestión de la educación sexual la coloca por fuera de una mirada integral e interdisciplinaria, situándose desde una perspectiva 
Revista de la Escuela de Ciencias de la Educación, año 14, nRo. 13, vol. 1, enero a junio de 2018. Páginas 105-126. ISSN 1851-6297 (DESDE DICIEMBRE DE 2006 A DICIEMBRE DE 2017). ISSN 2362-3349 (EN LiNeA). PARA UN ABORDAJE TRANSVERSAL DE LA ESI EN EL ESPACIO CURRICULAR DE FORMACIÓN ÉTICA Y CIUDADANA: REFLEXIONES SURGIDAS DESDE EL TRABAJO en formación docente. Melina Mailhou y Virginia Sabao Dominguez.

exclusivamente normativista. Transmitiendo una representación acerca de los niños, niñas y adolescentes, como sujetos vulnerables y posibles objetos de la violencia de terceros (adultos).

\section{c. En relación con las identidades y las diversidades}

En la presentación del NAP se apela a la sexualidad en tanto indicador de procesos de identificación:

La comprensión de la sexualidad humana a partir de su vínculo con la afectividad y los diferentes sistemas de valores y creencias: el encuentro con los otros/as, la pareja, el amor como apertura al otro/a, el cuidado mutuo en las relaciones afectivas. (NAP FEyC, 2011, p.8).

A su vez, los ítems de contenidos que mencionan la cuestión de la sexualidad como parte de la constitución de identidades y se hallan en el primer año en el DCESO son:

las interrelaciones entre diversidad sociocultural y desigualdad social y económica, identificando representaciones hegemónicas de género, orientación sexual, nacionalidad, etnia, religión, ideología y generación.

El reconocimiento de las diferentes temáticas acerca del género, con el propósito de superar visiones esencialistas y naturalizadas sobre los roles masculinos y femeninos, y de superar la reproducción de las relaciones jerárquicas entre los géneros. (DCESO, 2014, p. 18).

Y los ítems que se mencionan para segundo año en el DCESO son los que siguen:

análisis crítico de los roles de género a través de la historia hasta la actualidad. Reconocimiento de miradas estereotipadas. Reflexión acerca de las situaciones de igualdad o de falta de equidad de trato y de oportunidades en relación con el género y la orientación sexual en los diferentes ámbitos (la escuela, el hogar, el trabajo, la política, el deporte, entre otros).

El análisis de las representaciones sociales sobre los modelos corporales y estéticos identificando estereotipos y su incidencia en la construcción de identidades juveniles. Análisis y relación de estas identidades con los medios masivos de comunicación.

La comprensión de la sexualidad humana a partir de su vínculo con la afectividad y los diferentes sistemas de valores y creencias. (DCESO, 2014, pp. 20-21).

En este sentido, se observa que tanto en la propuesta de NAP como en el DCESO hay una apelación a la pareja, el amor y el cuidado mutuo. Aunque a su vez, se presenta un silencio que entendemos como currículum nulo respecto de la relación entre sexualidad y deseo. Temática que sin embargo, se 
Revista de la Escuela de Ciencias de la Educación, año 14, nRo. 13, vol. 1, enero a junio de 2018. Páginas 105-126. ISSN 1851-6297 (DESDE DICIEMBRE DE 2006 A DICIEMBRE DE 2017). ISSN 2362-3349 (EN LiNEA). PARA UN ABORDAJE TRANSVERSAL DE LA ESI EN EL ESPACIO CURRICULAR DE FORMACIÓN ÉTICA Y CIUDADANA: REFLEXIONES SURGIDAS DESDE EL TRABAJO en formación docente. Melina Mailhou y Virginia Sabao Dominguez.

expresa claramente en los Lineamientos Curriculares como tópico a trabajar en la articulación entre la FEyC y ESI.

También se destaca que en segundo año, el DCESO menciona por vez primera, la existencia de orientaciones sexuales, lo cual permite incluir junto a una perspectiva de género, una mirada acerca de la diversidad y/o heterogeneidad de las experiencias sexuales y su relación con la construcción de identidades.

\section{d. El cuarto eje:}

En la denominación planteada por el DCESO, el eje tiene algunas diferencias respecto de la propuesta del NAP para FEyC (En relación a una ciudadanía participativa).

En el título escogido para el eje cuatro como en los ítems de contenidos que se incluyen, hay una variación significativa respecto al NAP. Esta variación remite a la inclusión del análisis de lo social como organización. Es decir a una opción por el estudio organizacional/funcional-estructuralista de las relaciones sociales. Y a una inclusión de los conceptos familia, sociedad, persona y comunidad para el primer año; y de las nociones de familia, sociedad, sociedad de masas y organizaciones sociales para el segundo año. Así, la denominación del eje en primer año es: En relación con la organización social y una ciudadanía participativa. Y en segundo año es: En relación con la organización social y una ciudadanía responsable.

Aparece una constitución tensional entre participación y responsabilidad que no se encuentra así expresada en la propuesta de NAP. Y a su vez, si bien no se presenta ninguna explicitación que permita remitir algún ítem de contenido a algún tópico de ESI, resulta llamativo el carácter general desde el cual se refieren los fenómenos organizacionales. Ya que, en los ítems anteriores, las perspectivas de la diversidad y la desigualdad han sido intermitentemente incluidas con una sistemática referencia a la historicidad de los problemas sociales, cuestión que desaparece en este último eje.

En este sentido, la inclusión de la noción de "familia" (12) requeriría de una más pormenorizada identificación respecto a cómo pensarla incluyendo la perspectiva de ESI, ya que se opta por una mención con tonalidad general-abstracta, dónde no se propone un análisis histórico/estructural/cultural sobre la cuestión, ni se mencionan las perspectivas de la desigualdad y la diversidad para el tratamiento de tal tópico.

De este modo, entendemos que en el ítem cuarto que menciona temáticas nuevas y propias que se incluyen en la interpretación jurisdiccional sobre las prioridades a tener en cuenta en el trabajo de la FEyC, se hace presente una importante tensión respecto de las miradas recortadas en los tres ítems iniciales. Por un lado, se establece un examen deshistorizado y deshistorizante de los tópicos que se abordan y por otro, se introduce la cuestión de la familia 
Revista de la Escuela de Ciencias de la Educación, año 14, nRo. 13, vol. 1, enero a junio de 2018. Páginas 105-126. ISSN 1851-6297 (DESDE DICIEMBRE DE 2006 A DICIEMBRE DE 2017). ISSN 2362-3349 (EN LiNEA). PARA UN ABORDAJE TRANSVERSAL DE LA ESI EN EL ESPACIO CURRICULAR DE FORMACIÓN ÉTICA Y CIUDADANA: REFLEXIONES SURGIDAS DESDE EL TRABAJO en formación docente. Melina Mailhou y Virginia Sabao Domínguez.

(borrando por completo cualquier rastro de ESI en la enseñanza de la organización familiar).

Encontramos entonces, que la interpretación propuesta por parte de la jurisdicción de Santa Fe, incluye una matriz de análisis que tiene que ver con las apuestas interpretativas incluidas en diseños curriculares de FEyC del pasado, dónde las miradas normativista, positivista y funcional-estructuralista se articulaban en una hegemonización de la enseñanza de la ciudadanía como proceso de in-corporación de normas, sujeción a las mismas y adaptación funcional al orden social. (13). Y dónde la Ética y el Derecho dominaban la tarea con una presencia auxiliar de la sociología funcional-estructuralista para la elaboración de un empirismo reificador de la realidad existente. Sin embargo, la FEyC se postula hoy desde la lógica de los NAP como un espacio curricular multidisciplinar dónde la ética como disciplina filosófica, las ciencias de la cultura, las ciencias políticas y el derecho estarían interviniendo en forma equivalente respecto de sus legitimidades, aunque en una necesaria tensión, yuxtaposición e incluso oposición en relación a cada tópico problematizado.

\section{Reflexiones finales}

Interesa entonces resaltar las posibilidades que otorga el espacio curricular de FEyC para una articulación con la ESI. Es importante recordar que estos dos espacios del curriculum tienen en común la configuración de abordajes multi-inter-transdisciplinarios y desde una perspectiva de la complejidad, por lo que se vuelven pertinentes los enlaces de temas y de enfoques que se priorizan en ambos.

Pero más allá de estas efectivas posibilidades curriculares, desde una lectura preliminar de los Lineamientos Curriculares para ESI (2008), los NAP de FEyC (2011) y el DCESO (2014), podemos identificar desarticulaciones y tensiones entre las definiciones diversas de la ESI y de la ciudadanía que denotan distintas concepciones estatales acerca de los problemas mencionados.

En primer lugar, se observa un carácter más complejo y articulado del abordaje de la ESI en el documento de 2008. Allí aparece claramente explicitada la necesidad de relacionar distintos espacios curriculares con la enseñanza de la educación sexual integral; donde perspectivas socioculturales e históricas, psicológicas, jurídicas y biológicas se entrelazarían en función de un tratamiento integral de la experiencia sexuada de los/las agentes sociales. Sin embargo, cuando se observan los lineamientos para FEyC, aparece una homologación casi exclusiva entre este campo y la disciplina del Derecho. Por lo cual, lo que se gana en interdisciplina para el encuadre de la ESI, se pierde en la concepción que el documento establece para el de la ciudadanía.

En segundo lugar se indica en los NAP de FEyC ya desde la educación primaria que: 
Revista de la Escuela de Ciencias de la Educación, año 14, nRo. 13, vol. 1, enero a junio de 2018. Páginas 105-126. ISSN 1851-6297 (DESDE DICIEMBRE DE 2006 A DICIEMBRE DE 2017). ISSN 2362-3349 (EN LINEA). PARA UN ABORDAJE TRANSVERSAL DE LA ESI EN EL ESPACIO CURRICULAR DE FORMACIÓN ÉTICA Y CIUDADANA: REFLEXIONES SURGIDAS DESDE EL TRABAJO en formación docente. Melina Mailhou y Virginia Sabao Dominguez.

Los ejes se diferencian atendiendo a las disciplinas que integran el área: la ética como disciplina filosófica, las ciencias de la cultura, las ciencias políticas y el derecho. (NAP, Educación Primaria, Primer Ciclo. 2011, p.61).

Esta concepción implica, la afirmación de un enfoque multidisciplinario acerca del ethos y la ciudadanía, aunque a su vez, aparece un planteamiento de los problemas o tópicos a tratar en haciendo mención a una división del trabajo que distingue los temas/problemas a analizar según campos de conocimiento diferenciados. Propone una mirada segmentada sobre las temáticas, pensando cada eje de contenido como objeto/problemática propia de una episteme particular (la Ética, las Ciencias de la cultura, las Ciencias políticas o el Derecho). Así el énfasis en la cesura para el tratamiento de los ejes articuladores dentro del espacio curricular, conspira contra el abordaje multidisciplinario y desde una perspectiva de la complejidad que se pretende emprender.

A su vez, en el DCESO se observa que no existe una real transversalizacion de la ESI en el espacio de FEyC sino que aparece también como un tema adicional, como sucede con la educación vial u otras temáticas a incluir. De hecho la propuesta metodológica refiere al diseño y ejecución de seminarios intensivos dentro del espacio curricular. Es decir, no se postula una perspectiva de tipo transversal tal como se sugiere en los Lineamientos Curriculares ESI.

Un elemento a tener en cuenta tiene que ver con la presencia de temas de ESI incluidos en un listado de otras problemáticas que tienen que ver con la discriminación y el estigma. Lo cual no ayuda a la identificación de los procesos discriminatorios basados en la clasificación de géneros en particular.

Existe en FEyC (NAP, DCESO) un apartado que refiere a la sexualidad como elemento fundante de la condición humana y enfatiza la cuestión de la afectividad como constitutiva de tales relaciones, aunque se soslaya completamente la cuestión del deseo en la constitución de la sexualidad (elemento que sí introducen los Lineamientos Curriculares ESI).

También llama la atención, que en el DCESO se incluyan los tópicos de la niñez y la adolescencia desde una concepción que hace énfasis en su carácter vulnerable, ubicándolas como posibles objetos del avasallamiento de terceros. Consecuentemente con esta mirada, aparecen abordajes estrictamente jurídicos que se centran en el maltrato infantil, el abuso sexual y la trata de personas (delitos contra la integridad de los niños/as y adolescentes); así como en perspectivas de igualdad ante la ley de hombres y mujeres con heterogéneas formas de orientación sexual.

En el eje cuatro de FEyC del DCESO, se incluyen acríticamente las nociones de persona, familia y organización social, incorporándose así una tensión. En el cierre de la propuesta parece borrarse con el codo lo escrito con la mano. Aunque también podríamos pensar que la misma está habitada por una mirada paradojal sobre la ciudadanía, la subjetividad, el género, la sexualidad 
Revista de la Escuela de Ciencias de la Educación, año 14, nRo. 13, vol. 1, enero a junio de 2018. Páginas 105-126. ISSN 1851-6297 (DESDE DICIEMBRE DE 2006 A DICIEMBRE DE 2017). ISSN 2362-3349 (EN LiNEA). PARA UN ABORDAJE TRANSVERSAL DE LA ESI EN EL ESPACIO CURRICULAR DE FORMACIÓN ÉTICA Y CIUDADANA: REFLEXIONES SURGIDAS DESDE EL TRABAJO en formación docente. Melina Mailhou y Virginia Sabao Dominguez.

y la familia. Inevitablemente existen tesis opuestas y contradictorias en torno a la educación sexual y ciudadana en una convivencia conflictiva o de tenso equilibrio.

Finalmente, nos interesa registrar que, si bien existen perspectivas de la desigualdad y la diversidad, también se observa que el ingreso de nociones tales como: sí mismo, cuerpo, familia, sociedad, se establece de un modo acrítico, desde una mirada dónde la diversidad se encuentra deshistorizada y la desigualdad descontextualizada. En tal sentido, resulta llamativa la ausencia de la perspectiva antropológica y por lo tanto comparativa en el abordaje de tales problemas, que podría colaborar en una reposición de la historización de los procesos de homogeneización cultural operados en torno al cuidado de sí mediante los aparatos de salud y educación estatales. Así como a la identificación de las condiciones de desigualdad social en la reproducción de diversos grupos en contextos heterogéneos concretos. Estamos pensando en la necesidad de articular la perspectiva de género con una mirada decolonial, incluir análisis en torno a la etnogénesis en pueblos originarios de la Argentina actual y reconocer qué contenidos adquieren las nociones trabajadas en ESI para ellos (14), ya que como indica Nacach (2015),

La alteridad ha irrumpido en el marco de los Estados de Derecho como una narrativa otra que interpela, inquietante y extraña, desde los márgenes. Paralelamente, ha sido y aún hoy es imaginada a partir de representaciones tejidas históricamente que han permeado los sentidos sociales que habitan también en el Estado y sus agentes. (Nacach, 2015, p. 130).

Resulta ilustrativo indicar entonces, que tanto en la propuesta de los lineamientos nacionales como provinciales de la ESI, se reconocen sesgos que implican una serie de supuestos vinculados a la sexualidad como experiencia urbana. En que los conocimientos y saberes de pueblos originarios o de espacios rurales se ignoran o ingresan como no-saberes, incorrecciones o supersticiones contrarias a la ciencia. (15).

Así, resultaría recomendable explorar en la configuración de la enseñanza de nivel primario y secundario de Bolivia, para observar cómo se intenta sortear el problema de la negación de los saberes de las comunidades preexistentes a la constitución del Estado mediante la inclusión de contenidos específicos. (16).

En este sentido, volvemos a hacer mención, sobre la importancia que tienen los exámenes medicalizantes, correctivos y normalizadores en la propuesta de ESI. Ligados a una concepción cientificista y positivista sobre la experiencia sexual que sin embargo se tensionan con abordajes en torno a la cuestión de los procesos de identificación de los sujetos y los grupos en situación/contexto desde una perspectiva de género. Es decir, una convivencia compleja de lentes universalistas y particularistas en la ojeada que se realiza 
Revista de la Escuela de Ciencias de la Educación, año 14, nRo. 13, vol. 1, enero a junio de 2018. Páginas 105-126. ISSN 1851-6297 (DESDE DICIEMBRE DE 2006 A DICIEMBRE DE 2017). ISSN 2362-3349 (EN LiNEA). PARA UN ABORDAJE TRANSVERSAL DE LA ESI EN EL ESPACIO CURRICULAR DE FORMACIÓN ÉTICA Y CIUDADANA: REFLEXIONES SURGIDAS DESDE EL TRABAJO en formación docente. Melina Mailhou y Virginia Sabao Dominguez.

en torno a la ESI, que sin embargo mantienen en un estado invisibilizado el conjunto de experiencias y concepciones que gran parte de la población argentina posee en torno a los problemas que la ley incluye.

Precisamente, en este sentido retomamos la recomendación de Nacach (2015):

Tensar la idea de lo universal y lo particular, entendiendo que la especificidad -en este caso la identidad étnica- no corroe la idea de lo común sino que aporta a la comprensión de la diversidad en tanto riqueza, nos permitirá dejar de ver a los otros (todos los mundos otros) como una incógnita o como extrañeza, como parte de algo incomprensible y por lo mismo inabordable. Restituir complejidad a los procesos sociohistóricos que atravesaron los pueblos indígenas y que marcan su identidad presente es algo que excede a la ESI pero que encuentra en ella una excusa más que interesante para indagar con ellos sobre los sentidos de las prácticas sociales históricamente situadas. (Nacach, 2015, p. 131).

Quizás sería necesario barajar y dar de nuevo. Comenzando por pensar a la ESI de una manera transdisciplinaria, que incluya saberes, experiencias y sensibilidades de diversos grupos que componen la población de la Argentina en torno al parentesco, la sexualidad, la afectividad y el cuidado de sí, sin dar por sentados sus contenidos, operar bajo generalizaciones apriorísticas y clasificaciones tales como: salud/enfermedad, normal/patológico, legal/ilegal. Y a partir de esta reconstrucción analítica de enfoques múltiples en el campo social, reconsiderar los enfoques disciplinares fundamentales a articular en la problematización de la experiencia sexuada.

Esto, implicaría entonces un abordaje cada vez más colegiado/participativo, en el que los diversos grupos de identidad participen legítimamente y no se jerarquicen o autoricen algunas voces por sobre otras. Un trabajo orientado hacia la identificación de la diversidad de formas en que se experimenta la sexualidad y las tensiones existentes entre algunas de tales concepciones particulares.

Las críticas desarrolladas a lo largo de este artículo quieren potenciar e incluso sostener y defender una herramienta fundamental para el trabajo docente como es la ESI, ya que si bien la ley 26.150 se encuentra vigente, sabemos de la reorientación de las actuales políticas nacionales en torno al problema, que implican desmantelamiento de programas, desfinanciamiento y reorganización de la capacitación.(17) 
Revista de la Escuela de Ciencias de la Educación, año 14, nRo. 13, vol. 1, enero a junio de 2018. Páginas 105-126. ISSN 1851-6297 (DESDE DICIEMBRE DE 2006 A DICIEMBRE DE 2017). ISSN 2362-3349 (EN LiNEA). PARA UN ABORDAJE TRANSVERSAL DE LA ESI EN EL ESPACIO CURRICULAR DE FORMACIÓN ÉTICA Y CIUDADANA: REFLEXIONES SURGIDAS DESDE EL TRABAJO en formación docente. Melina Mailhou y Virginia Sabao Dominguez.

\section{Notas bibliográficas:}

(1) "Los ejes se diferencian atendiendo a las disciplinas que integran el área: la ética como disciplina filosófica, las ciencias de la cultura, las ciencias políticas y el derecho". (NAP, Educación Primaria, Primer Ciclo. 2011, p.61).

(2) Spadaro María "La coeducación en la enseñanza de la filosofía: una deuda de género". En Cerletti A. (comp) La enseñanza de la filosofia en perspectiva, Buenos Aires, EUDEBA, 2009.

(3) Un antecedente importante en cuanto a reflexiones surgidas desde la práctica docente lo constituye el trabajo de Beatriz Argiroffo y Elvira Scalona (compiladoras) (2016). Educación Sexual Integral y enseñanza de las Ciencias Sociales. Enfoques, sujetos y objetos para su abordaje en la escuela secundaria. Rosario: el autor.

(4) Con respecto al carácter multidisciplinario sumativo del enfoque de la educación sexual, vid. DCESO pp. 111-114.

(5) Cf. Ibid.

(6) Vid. DCESO, p. 16 donde se define la Ética desde una perspectiva tradicional como reflexión sobre la distinción entre lo bueno y lo malo.

(7) Cf. Ibid.

(8) Vid. DCESO, pp. 10-11.

(9) Lo que indica una inclusión de la reflexión ética como dispositivo conservador de valores consensuales que tiende a la resolución-neutralización de contradicciones/ tensiones del campo social.

(10) Bourdieu, indica que la característica propia del discurso jurídico, genera efectos de neutralización y universalización: "El efecto de neutralización... para resaltar la impersonalidad de la enunciación normativa y para constituir al enunciante en sujeto universal, a la vez imparcial y objetivo. ...el efecto de universalización ... para expresar la generalidad y la omnitemporalidad de la norma jurídica; la referencia a valores trans-subjetivos, que presuponen la existencia de un consenso ético" (Bourdieu, 2000, p.166).

(11) Sin olvidar, que sólo en el caso de esta propuesta, se busca un ejercicio argumentativo racional que tienda a la identificación de falacias.

(12) Vid. DCESO, p.18 y p. 21.

(13) Cf. Ibid.

(14) Existen diversos trabajos que podemos recomendar como referencias de lectura sobre la cuestión: Nacach, Gabriela (2015). "Los mensajes de la capacitación: Un posible abordaje de la Educación Sexual Integral en contextos indígenas", Etnografías Contemporáneas 1. Menna, Fabiana; Kelly, Silvia María; Liziardi, Carina; Benítez, Liliana (comps.) (2014). Derechos sexuales y reproductivos de las mujeres wichí. Buenos Aires, CICCUS. Tola, Florencia (2012). Yo no estoy solo en mi cuerpo. Cuerpos-personas múltiples entre los qom (tobas) del Gran Chaco. Buenos Aires, Biblos/ Culturalia. Segato, Rita (2011). "Género y colonialidad: en busca de claves de lectura y de un vocabulario estratégico decolonial”, en Quijano, Aníbal y otro. La cuestión descolonial. Lima, Universidad Ricardo Palma. Gómez, Mariana (2011). "Tensiones espaciales y ansiedades sexuales: memorias sobre la juventud 
Revista de la Escuela de Ciencias de la Educación, año 14, nRo. 13, vol. 1, enero a junio de 2018. Páginas 105-126. ISSN 1851-6297 (DESDE DICIEMBRE DE 2006 A DICIEMBRE DE 2017). ISSN 2362-3349 (EN LiNEA). PARA UN ABORDAJE TRANSVERSAL DE LA ESI EN EL ESPACIO CURRICULAR DE FORMACIÓN ÉTICA Y CIUDADANA: REFLEXIONES SURGIDAS DESDE EL TRABAJO en formación docente. Melina Mailhou y Virginia Sabao Dominguez.

de mujeres qom", en Vázquez Laba, Vanesa: Feminismos sexualidades y religiones en mujeres subalternas. Buenos Aires, Colección Religión, Género y Sexualidad. Citro, Silvia (2008). "Creando una mujer. Ritos de iniciación femenina y matriz simbólica de los géneros entre los tobas takshik", en Hirsch, Silvia: Mujeres indígenas en la Argentina: cuerpo, trabajo y poder. Buenos Aires, Biblos, pp. 27-58. Briones, Claudia (2005). Cartografías Argentinas: políticas indígenas y formaciones provinciales de alteridad. Buenos Aires, Antropofagia.

(15) En este sentido, resulta interesante mencionar el estudio de Canessa, A (2007), en el cual identifica a las instituciones del Estado boliviano como la Escuela y el Ejército, en tanto espacios que adquieren una importante eficacia en la introducción de representaciones asimétricas (raciales y de género) en sujetos de comunidades rurales, dónde ciertas concepciones de complementariedad de géneros están presentes en forma previa a la intervención estatal. Escolarizarse ofrece a las guaguas ciudadanía en la nación, al darles acceso a las modales y el idioma metropolitanos, pero con el costo de introducir representaciones hegemónicas de raza y género. (p. 12).

(16) Para un análisis detallado consultar: Reglamento de Gestión Curricular del Subsistema de Educación Regular. Ministerio de Educación de Bolivia. 2013. PROFOCOM. Comprendiendo la estructura curricular. Ministerio de Educación. Estado Plurinacional de Bolivia. 2012.

(17) En un contexto en que la ESI requiere de mayor participación activa de los diversos grupos que configuran colectivos interesados en visibilizar sus concepciones y prácticas en torno a la sexualidad y las relaciones de género, el Decreto 690/2017 del 05/09/2017 indica la disolución del Consejo Nacional de Mujeres (organismo colegiado y participativo) para ser reemplazado por el Instituto Nacional de Mujeres (INAM): "de manera de potenciar los lineamientos de la Ley $N^{\circ} 26.485$, profundizando un modelo inclusivo y de participación con equidad, suprimiendo en consecuencia el citado Consejo". (Decreto 698/2017). Es decir, que en una coyuntura que reclama mayor participación colegiada en el ámbito de la educación sexual integral y de la prevención de la violencia hacia las mujeres, el Estado Nacional decide disolver el organismo que reúne tales condiciones para sustituirlo por una institución del estado dónde la participación organizaciones de la sociedad civil no constituye precondición.

\section{Referencias bibliográficas}

- Bilinkis, M. (2013). La educación sexual en disputa: una aproximación a los debates parlamentarios de la Ley Nacional de Educación Sexual Integral. Boletín de Antropología y Educación. pp. 27-29. Año 4 - № 05.

- Boas, F. (1964). Fundamentos de antropología cultural. Buenos Aires: Ed. SOLAR y HACHETE.

- Bourdieu, P. (2000). La fuerza del derecho, Elementos para una sociología del campo jurídico". En: García Inda (comp.). Poder, derecho y clases sociales. Bilbao: Ed. Palimpesto. 
Revista de la Escuela de Ciencias de la Educación, año 14, nRo. 13, vol. 1, enero a junio de 2018. Páginas 105-126. ISSN 1851-6297 (DESDE DICIEMBRE DE 2006 A DICIEMBRE DE 2017). ISSN 2362-3349 (EN LíNEA). PARA UN ABORDAJE TRANSVERSAL DE LA ESI EN EL ESPACIO CURRICULAR DE FORMACIÓN ÉTICA Y CIUDADANA: REFLEXIONES SURGIDAS DESDE EL TRABAJO en formación docente. Melina Mailhou y Virginia Sabao Domínguez.

- Canessa, A. (2007). El indio desde adentro, el indio desde afuera: ciudadanía, raza y sexo en una comunidad boliviana. En: La manzana de la discirdia. pp. 7-23. Año $2-\mathrm{N}^{\circ} 04$.

- Foucault, M. (2000). Historia de la sexualidad. La voluntad de saber. México: Siglo XXI.

- Habermas, J. (2005). Política deliberativa: Un concepto procedimental de la democracia. En: Factibilidad y validez. Madrid: Ed. Trotta.

- Herrera, D. y Rosemberg, D. (2012). El desafío de la educación sexual. Cuadernos de discusión \# 4. UNIPE: Editorial Universitaria. La Plata.

- Herskovist, M. (1968). El hombre y sus obras. D.F.: FCE.

- Kluckhohn, C. (1981). Antropología. D.F.: FCE.

- Lefort, C. (1981). ¿Permanece lo teológico político?. Buenos Aires: Ed: Hachete.

- Mead, M. (1973). Sexo y temperamento en tres sociedades primitivas. Barcelona: Ed. Laia.

- Mouffe, C. (2014). Agonística: Pensar el mundo políticamente. Buenos Aires: FCE.

- Nacach, G. (2015). Los mensajes de la capacitación: Un posible abordaje de la Educación Sexual Integral en contextos indígenas. En: Etnografías Contemporáneas 1 (1). pp.116-136.

- Spadaro, M. (2009). La coeducación en la enseñanza de la filosofía: una deuda de género. En: Cerletti, A. (comp). La enseñanza de la filosofia en perspectiva. Buenos Aires: EUDEBA.

\section{Fuentes Documentales:}

- Decreto 698/2017. Creación de la Agencia Nacional de Discapacidad.

- Ley N 26.150 de Educación Sexual Integral.

- Lineamientos Curriculares para la ESI, 2008. Ministerio de Educación de la Nación. Argentina.

- $\quad \mathrm{NAP}, \mathrm{FEyC}$, 2011. Ciclo Básico ESO, CFE.

- $\quad$ NAP, FEyC, 2011. Ciclo Básico ESO, Educación Primaria. CFE.

- Núcleos Interdisciplinarios de Contenidos. La educación en acontecimientos. Documento de Desarrollo Curricular. Ministerio de Educación de la Provincia de Santa Fe. 2016.

- PROFOCOM. Comprendiendo la estructura curricular. Ministerio de Educación. Estado Plurinacional de Bolivia. 2012.

- Reglamento de Gestión Curricular del Subsistema de Educación Regular. Ministerio de Educación de Bolivia. 2013.

- Res. 2630/14. Diseño Curricular para la Educación Secundaria. Ministerio de Educación de la Provincia de Santa Fe. 